\title{
Linkage analysis with chromosome 15q11-13 markers shows genomic imprinting in familial Angelman syndrome
}

\author{
E J Meijers-Heijboer, L A Sandkuijl, H G Brunner, H J M Smeets, \\ A J M Hoogeboom, W H Deelen, J O van Hemel, M R Nelen, D F C M Smeets, \\ M F Niermeijer, D J J Halley
}

\begin{abstract}
Angelman syndrome (AS) and PraderWilli syndrome (PWS) have become the classical examples of genomic imprinting in man, as completely different phenotypes are generated by the absence of maternal (AS) or paternal (PWS) contributions to the q11-13 region of chromosome 15 as a result of deletion or uniparental disomy. Apparently, most patients are sporadic cases. The genetic mechanism underlying familial AS has remained enigmatic for a long time. Recently, evidence has been emerging suggesting autosomal dominant inheritance of a detectable or undetectable defect in a gene or genes at 15q11-13, subject to genomic imprinting. The present report describes an unusually large pedigree with segregation of AS through maternal inheritance and apparent asymptomatic transmission through several male ancestors. Deletion and paternal disomy at 15q11-13 were excluded. However, the genetic defect is still located in this region, as we obtained a maximum lod score of 5.40 for linkage to the GABA receptor locus GABRB3 and the anonymous DNA marker D15S10, which have been mapped within or adjacent to the $A S$ critical region at 15q11-13. The size of the pedigree allowed calculation of an odds ratio in favour of genomic imprinting of $9 \cdot 25 \times 10^{5}$. This family illustrates the necessity of extensive pedigree analysis when considering recurrence risks for relatives of AS patients, those without detectable deletion or disomy in particular.
\end{abstract}

( $\mathcal{F}$ Med Genet 1993;30:853-7)

E J Meijers-Heijboer

L A Sandkuijl

A J M Hoogeboom

W H Deelen

J O van Hemel

M F Niermeijer

D J J Halley

Department of Human Genetics, University Hospital

Nijmegen, The

Netherlands.

Hetherlands.

H J M Smeets

$M$ R Nelen

D F C M Smeets

Correspondence to Dr Meijers-Heijboer

Received 11 August 1992 Accepted 2 September 1992 genetically visible deletion or rearrangeme of chromosome $15 q 11-13^{24}$ and 75 to $80 \%$ of the patients have molecular deletions. ${ }^{25}$ The deletion always involves the maternally inherited chromosome $15 .^{26} \mathrm{~A}$ small percentage of non-deletion AS patients lack the maternal 15q11-13 region as a result of paternal disomy. ${ }^{278}$

The same cytogenetically defined region of chromosome 15 is involved in Prader-Willi syndrome (PWS). ${ }^{9}$ PWS is phenotypically very different from $A S$ as PWS patients display infantile hypotonia, childhood hyperphagia and obesity, mental retardation, and hypogenitalism. ${ }^{10}$ In contrast to AS, in PWS the deletion of chromosome 15q11-13 is of paternal origin, ${ }^{411}$ whereas uniparental disomies are of maternal origin. ${ }^{12}$ The different parental origins of $15 \mathrm{q}$ deletions and disomies resulting in different phenotypes indicate that genes in this region show differential expression on maternal versus paternal chromosomes (genomic imprinting). The report of a family with a chromosomal translocation involving chromosome 15 leading to unbalanced translocations with deletions in $15 q$ in the offspring supports the role of genomic imprinting in both syndromes, as identical unbalanced karyotypes in the offspring either produced AS or PWS, depending on the sex of the transmitting parent. ${ }^{13}$

Recently, molecular analysis showed that the smallest regions of overlap (SRO) of the deletions in both syndromes are distinct, the SRO of AS residing between D15S11 and $D 15 S 10^{14}$ and the SRO of PWS between D15S9 and D15S11. ${ }^{15}$ This suggests that different genes are involved in the aetiology of both syndromes. It is conceivable that a defect in inhibitory neurotransmission mediated by the $\mathrm{GABA}_{\mathrm{A}}$ receptor plays a role in the pathogenesis of AS. ${ }^{16}$ Therefore, the $\mathrm{GABA}_{\mathrm{A}}(\gamma-$ aminobutyric acid) $\beta 3$ subunit receptor (GABRB3) gene, which has recently been localised to the SRO of AS, has been proposed as a candidate gene for AS. ${ }^{16} 17$

The vast majority of patients with AS are apparently sporadic cases. Several familial AS patients have been reported and, unlike sporadic cases, detectable abnormalities of chromosome $15 q$ are rare. ${ }^{2}$ Since most of the familial AS patients are sibs, it has been hypothesised that AS may be inherited as an autosomal recessive trait. $^{18}$ However, Hamabe et $a l^{14}$ reported a family in which three AS sibs did show a deletion of $15 \mathrm{q}$, which they shared with their healthy mother and grandfather. Recently, Wagstaff et al ${ }^{19}$ reported three unaffected sisters with affected offspring without 
detectable abnormalities involving $15 \mathrm{q}$, most likely representing autosomal dominant inheritance and genomic imprinting at 15q. In this family a maximum lod score of 2.91 at $\theta=0.00$ with $G A B R A 5(C A)-1^{19}$ was obtained. Marker $G A B R A 5(C A)-1$ was reported to be localised distal to the SRO of AS. ${ }^{19}$

We present a family of exceptional size as it spans five generations with AS occurring in several sibships. Maternal inheritance was apparent in each case. Asymptomatic transmission through several male ancestors could be inferred from the pedigree. Cytogenetic analysis was performed to study chromosomal rearrangements in the patients and their mothers. Molecular and statistical analyses were applied to investigate subtle deletions or uniparental disomy involving 15q, linkage between $A S$ and markers on $15 \mathrm{q}$, and to determine the mode of inheritance.

\section{Materials and methods PATIENTS}

The pedigree of a five generation Caucasian family with eight AS patients is shown in fig 1 . Seven of the patients showed typical features of AS, including severe mental retardation, absent speech, and frequent laughter and smiling (table 1). Before this family presented to us, the only patient in generation III (fig 1) had died at the age of 53 years. Her medical records and photographs confirmed that she was also affected with AS. Patient V.3 was seen at the age of 10 months and all other patients and family members investigated were older than 10 years. Seven patients had EEG abnormalities, while two patients, IV.10 and III.x, had seizures. Strabismus was seen in patients V.1, V.3, IV.6, and III.x. Fig $2 \mathrm{~A}$ and B show the development of the facial dysmorphism in patient IV.10. Fig 2C shows patient V.1 at the age of 8 years. There was no other family history of congenital malformations, developmental delay, or epilepsy. Consanguineous marriages were not recorded.

\section{CYTOGENETIC ANALYSIS}

Chromosome analysis on lymphocytes of patients V.1, V.3, IV.6, IV.7, IV.15, and IV.16 was performed using standard techniques. High resolution GTG banding was applied to study one of the patients (IV.10) in more detail. In order to enable detection of a familial translocation of chromosome 15 in its unbalanced form, the patients' mothers, IV.2, III.8, III.10, and III.13, were also karyotyped.

\section{DNA ANALYSIS}

Blood samples for DNA analysis were collected from 38 subjects, as indicated in fig 1, representing three generations and including all available nuclear families with affected offspring. DNA from leucocytes was isolated according to Miller et al. ${ }^{2021}$ Dinucleotide (CA) repeat markers at $G A B R B 3$ and $D 15 S 10$ were analysed using radiolabelled PCR conditions essentially as previously described. ${ }^{2122}$ RFLP

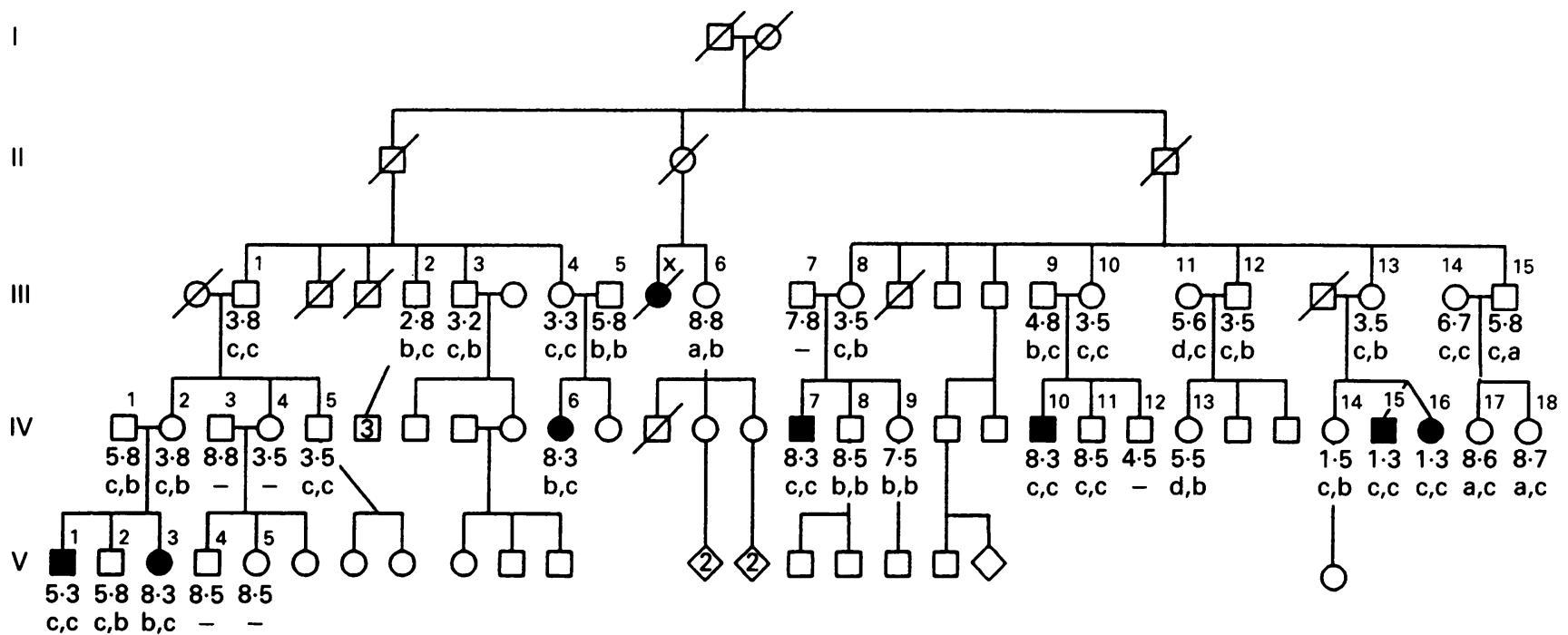

Figure 1 Family pedigree. Filled symbols= affected. All numbered subjects were typed for GABRB3-CA (alleles 1-8) and D15S10-CA (alleles $a-d)$, as well as for RFLPs at D15S18, D15S13,D15S9, D15S11, D9S10, and D8S12 (not shown). III.x is the dead patient.

Table 1 Clinical symptoms in the eight AS patients.

\begin{tabular}{lcccccccc}
\hline & V.1 & V.3 & IV.6 & III.x & IV.7 & IV.10 & IV.15 & IV.16 \\
\hline Mental retardation & + & + & + & + & + & + & & + \\
Absent speech & + & + & + & + & + & + & + & + \\
Paroxysms of laughter & + & + & + & + & + & + & + & + \\
Abnormal gait & + & + & + & + & + & + & + & + \\
Seizures/EEG abnormalities & + & ND & + & + & + & + & + & + \\
Microcephaly & + & + & + & + & + & + & + & + \\
Brachycephaly & + & + & + & ND & + & + & + & + \\
Macrostomia & + & + & + & + & + & + & + & + \\
Prognathism & + & - & + & + & + & + & + & + \\
\hline
\end{tabular}

$+=$ present,$-=$ absent, $\mathrm{ND}=$ no data available 


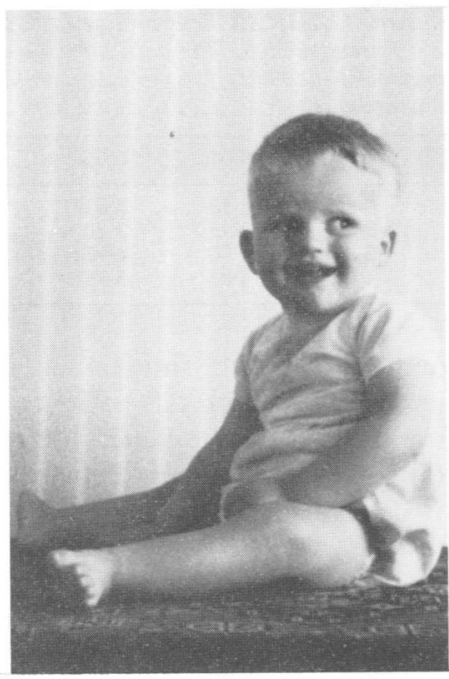

(A)

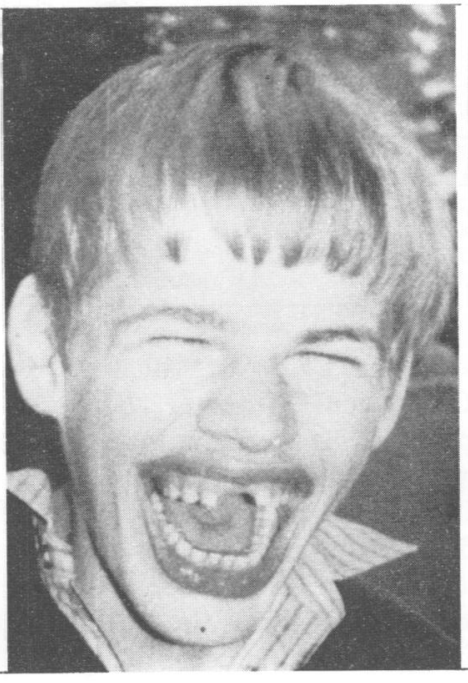

(B)

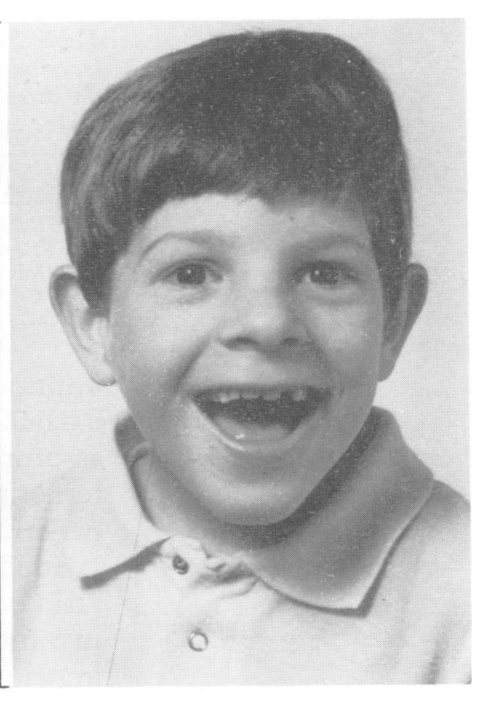

(C)

Figure 2 (A) Patient IV.10 at the age of 1 year. (B) The same patient at the age of 10 years, showing that facial dysmorphism evolves with time. (C) Patient V.1 aged 8 years.

analysis by Southern blotting and hybridisation using standard methods involved the following probes from 15q11-13: pIR39 (locus D15S18), pTD189.1 (D15S13), pML34 (D15S9), pIR4-3R (D15S11), pTD3-21 (D15S10), and pIR10-1 (D15S12). ${ }^{11}$

\section{LINKAGE ANALYSIS}

All linkage calculations were carried out with the MLINK option of the LINKAGE package of computer programs (version 5.03). ${ }^{23}$ Equal allele frequencies were assumed for the marker loci. The disease allele was given a frequency of 0.001 in the calculations, but it was assumed that only a single AS mutation segregated in this family. Calculations were carried out under two models: one with $50 \%$ penetrance for all subjects heterozygous for the autosomal dominant disease allele (absence of the disease phenotype in gene carriers is explained by random effects leading to $50 \%$ penetrance), and another model with complete penetrance for subjects who had inherited the disease allele from their mother, while paternal inheritance was assumed never to lead to expression of the mutation (absence of the disease phenotype in gene carriers is solely the effect of genomic imprinting). Odds for imprinting were calculated by comparing the maximum likelihood obtained under the model of imprinting and linkage with the maximum likelihood allowing for linkage but not for imprinting.

Subjects V.1, V.3, IV.6, III.x, IV.7, IV.10, IV.15, and IV.16 were considered to be affected, while all other family members were considered to be unaffected.

In the multipoint analysis the distance between $G A B R B 3$ and $D 15 S 10$ was fixed at $1 \mathrm{cM}$, based on the observation of a single recombination event between these loci in this pedigree. Recombination frequencies in males and females were assumed to be equal.

\section{Results}

CYTOGENETIC AND DNA ANALYSIS

Chromosome analysis showed normal karyotypes in all seven investigated patients and their mothers, with no cytogenetically visible deletions or translocations involving chromosome $15 q$.

DNA marker analysis showed heterozygosity at $G A B R B 3$ in all patients and at $D 15 S 10$ (CA repeat) in two patients (fig 1). Heterozygosity was also observed at D15S11 in the DNA of four patients when analysing the StyI and RsaI polymorphisms detected by IR4-3R (not shown). The hybridisation patterns obtained by Southern blotting using other RFLP markers from chromosome 15q11-13 were all in agreement with the presence of two alleles in the patients' DNA. Hence, we were unable to detect a deletion in the chromosomal region we investigated at the molecular level. Moreover, the heterozygous patterns at $G A B R B 3$ were of unequivocal biparental origin in five of the patients (fig 1). The maternal allele could also be distinguished with certainty in the four patients who were heterozygous at D15S11 (not shown). Assuming that one genetic defect segregates in this family this rules out paternal disomy at these loci as the cause of AS in this family.

\section{LINKAGE ANALYSIS}

We noticed that the same allele, numbered 3 in fig 1 , at $G A B R B 3$ was found in all seven patients investigated, their mothers, and the only living grandparent (III.1), whereas this allele was not observed in unaffected sibs of patients (fig 1). Next, we analysed whether the genetic defect in this family showed linkage to $G A B R B 3$ and $D 15 S 10$.

Table 2 summarises the lod scores obtained at different $\theta$, loci, and models. No recombinations between $A S$ and either $D 15 S 10$ or the $G A B R B 3$ locus were observed. A maximum 
Table 2 Lod scores between $A S$ and $C A$ repeats at the GABRB3 locus and at D15S10 at various recombination fractions. Lod scores were calculated assuming two different models: one with $50 \%$ penetrance of all heterozygotes for the disease allele (no imprinting), and one with complete penetrance for subjects who had inherited the disease allele from their mother, while paternal inheritance was assumed never to lead to expression of the mutation.

\begin{tabular}{|c|c|c|c|c|c|c|c|}
\hline & $\theta$ & 0.0 & 0.01 & 0.05 & 0.1 & 0.2 & 0.3 \\
\hline $\begin{array}{l}\text { GABRB3-CA } \\
D 15 S 10-C A \\
\text { GABRB3-CA+D15S10-CA with imprinting } \\
\text { GABRB3-CA+D15S10-CA without imprinting }\end{array}$ & & $\begin{array}{l}4 \cdot 28 \\
3 \cdot 01 \\
5 \cdot 40 \\
4 \cdot 09\end{array}$ & $\begin{array}{l}4 \cdot 18 \\
2 \cdot 95 \\
5 \cdot 30 \\
4 \cdot 03\end{array}$ & $\begin{array}{l}3 \cdot 81 \\
2 \cdot 70 \\
4 \cdot 91 \\
3 \cdot 75\end{array}$ & $\begin{array}{l}3 \cdot 36 \\
2 \cdot 37 \\
4 \cdot 40 \\
3 \cdot 39\end{array}$ & $\begin{array}{l}2.53 \\
1.67 \\
3.36 \\
2 \cdot 61\end{array}$ & $\begin{array}{l}1 \cdot 71 \\
0.93 \\
2 \cdot 26 \\
1.75\end{array}$ \\
\hline
\end{tabular}

multipoint lod score of 5.40 was obtained at $\theta=0.00$ between $A S$ and $G A B R B 3$ under the assumption of genomic imprinting. The alternative model assumed incomplete $(50 \%)$ penetrance instead of genomic imprinting and yielded a substantially lower lod score. The ratio between the maximum likelihoods of the two models determines the odds for genomic imprinting. Assuming complete linkage of the AS gene(s) with $D 15 S 10$ and $G A B R B 3$, an odds ratio in favour of imprinting of $9 \cdot 25 \times 10^{5}$ was calculated.

\section{Discussion}

The AS patients in the family reported here showed no evidence for a deletion or other chromosomal abnormality involving 15q1113. Paternal disomy of chromosome 15 was excluded as the cause of AS in this family. The maximum lod score of 5.40 for linkage with $G A B R B 3$ and $D 15 S 10$ provides strong evidence for a dominant mutation in 15q11-13. For the first time the role of genomic imprinting in familial AS could be established by statistical analysis, as an odds ratio of $9.25 \times 10^{5}$ in favour of genomic imprinting versus reduced penetrance was achieved. The pattern of imprinting in this family was such that no father passed on the disease, but only the females II.2, III.4, 8, 10, 13, and IV.2 (fig $1)$. The data indicate that the mutation causing AS in the eight family members originates from the male ancestor in generation I (fig 1). Apparently, the mutation was transmitted through as many as three generations by male carriers without any phenotypic expression. This fact is of great importance in view of the risk assessments for even distant female relatives, who may have a $50 \%$ risk of affected offspring depending on their position in the pedigree (fig 1).

The AS families reported by Hamabe $e t$ al ${ }^{14}$ and Wagstaff $e t a l^{19}$ are in agreement with the concept of a dominant mutation in one or more genes on 15q11-13 subject to imprinting as the cause of familial AS. Two other AS families have been reported ${ }^{24}$ that were investigated with DNA markers. In one case the affected sibs inherited the same maternal 15q11-13 region. The affected sibs in the other family inherited at least partly different maternal regions of 15q11-13, but a possible recombination event between the SRO of AS and the tested markers made the latter result inconclusive.

We conclude that the present molecular data from familial AS patients are either in favour of a dominant genetic defect on 15q11-13 with phenotypic expression depending on imprinting or do not contradict this hypothesis.
Further molecular genetic analysis of nondeletion AS patients will be essential to determine the gene(s) involved in the pathogenesis of AS. Our data do not contradict a possible role of the GABRB3 gene in the pathogenesis of AS as no recombinations were detected between $A S$ and this locus in the entire family.

The consistent lack of PWS in the offspring of the male carriers of an AS mutation adds to the evidence that distinct defects are involved in the aetiologies of AS and PWS. In our family non-penetrance as an explanation for the lack of PWS offspring was highly unlikely as multiple transmissions of the mutation occurred through male meioses without phenotypic expression of PWS.

The influence of genomic imprinting on the expression of mutation has been described in a growing number of human genetic diseases. ${ }^{25}$ Hereditary paraganglioma is another example where the role of genomic imprinting in the phenotypic expression in an extended family has been shown to be absolute. ${ }^{26}$

The recent implication of genomic imprinting in familial AS may complicate genetic counselling. In familial AS cases, an $\mathrm{X}$ linked mode of inheritance may falsely be deduced when the syndrome diagnosis is overlooked. On the other hand, imprinting may obscure the hereditary nature of the defect owing to the lack of phenotypic expression in offspring of male carriers. If a genetic defect at 15q11-13 and consequently genomic imprinting are a universal phenomenon in familial AS, unaffected sibs of AS patients would have no increased risk for AS in their offspring. Risk assessments for more distant female relatives of AS patients, particularly those without detectable genetic defects, would necessarily involve an extensive search of the pedigree for AS

We thank Dr B A van Oost for critical reading of the manuscript.

1 Angelman H. 'Puppet' children. Dev Med Child Neurol 1965;7:681-8.

2 Clayton-Smith J, Pembrey ME. Angelman syndrome. $f$ Med Genet 1992;29:412-5.

3 Fryburg JS, Breg WR, Lindgren V. Diagnosis of Angelman syndrome in infants. Am $\mathcal{F}$ Med Genet 1991;38:58-64.

4 Kaplan LC, Wharton R, Elias E, et al. Clinical heterogeneity associated with deletions in the long arm of chromosome 15: report of 3 new cases and their possible significance. Am $\mathfrak{F}$ Med Genet 1987;28:45-53.

5 Donlon TA. Similar molecular deletions on chromosome 15q11.2 are encountered in both Prader-Willi and Angelman syndromes. Hum Genet 1988;80:322-8.

6 Knoll JHM, Nicholls RD, Magenis RE, et al. Angelman and Prader-Willi syndrome share a common chromosome 15 deletion but differ in parental origin of the deletion. Am $\mathcal{F}$ Med Genet 1989;32:285-90.

7 Malcolm S, Clayton-Smith J, Nichols M, et al. Uniparental paternal disomy in Angelman's syndrome. Lancet 1991;337:694-7.

8 Smeets DFCM, Hamel BCJ, Nelen MR, et al. Prader-Willi syndrome and Angelman syndrome in cousins from a 15. $N$ Engl f Med 1992;326:807-11. 
9 Ledbetter DH, Riccardi VM, Aihart SD, et al. Deletions of chromosome 15 as a cause of the Prader-Willi syndrome. N Engl $f$ Med 1981;304:325-9.

10 Prader A, Labhart A, Willi H. Ein Syndrome von Adipositas, Kleinwuchs, Kryptorchismus und Oligophrenie nach as, Kleinwuchs, Kryptorchismus und Oligophrenie nach
myatonieartigem Zustand in Neugeborenenalter. Schweiz Med Wochenschr 1956;86:1260-1.

11 Nicholls RD, Knoll JHM, Glatt $\mathrm{K}$, et al. Restriction fragment length polymorphism within proximal $15 \mathrm{q}$ and their use in molecular cytogenetics and the Prader-Willi syndrome. Am $\mathcal{F}$ Med Genet 1989;33:66-77.

12 Nicholls RD, Knoll JHM, Butler MG, et al. Genetic imprinting suggested by maternal heterodisomy in nondeletion Prader-Willi syndrome. Nature 1989;342:281-5.

13 Hultén M, Armstrong S, Challinor P, et al. Genomic imprinting in an Angelman and Prader-Willi translocation family. Lancet 1991;338:638-9.

14 Hamabe J, Kuroki Y, Imaizumi K, et al. DNA deletion and its parental origin in Angelman syndrome patients. $A m \mathcal{F}$ $1991 ; 41: 64-8$.

15 Hamabe J, Fukushima Y, Harada N, et al. Molecular study of the Prader-Willi syndrome: deletion, RFLP, and phenotype analyses of 50 patients. Am $\mathcal{F}$ Med Genet 1991;41:54-63.

16 Wagstaff J, Knoll JHM, Fleming J, et al. Localization of the gene encoding the $\mathrm{GABA}_{\mathrm{A}}$ receptor $\beta 3$ subunit to the Angelman/Prader-Willi region of human chromosome 15. Am F Hum Genet 1991;49:330-7.

17 Saitoh S, Sugimoto T, Wagstaff J, et al. Familial Angelman syndrome caused by imprinted submicroscopic deletion encompassing $\mathrm{GABA}_{\mathrm{A}}$ receptor $\beta 3$-subunit gene. Lancet 1992;339:366-7.
18 Baraitser M, Patton M, Lam ST, et al. The Angelman (happy puppet) syndrome: is it autosomal recessive? Clin Genet 1987;31:323-30.

19 Wagstaff J, Knoll JHM, Glatt KA, et al. Maternal but not paternal transmission of 15q11-13-linked nondeletion Angelman syndrome leads to phenotypic expression Nature Genet 1992;1:291-4.

20 Miller SA, Dykes DD, Polesky HF. A simple salting out procedure for extracting DNA from human nucleated cells. Nucleic Acids Res 1988;16:1214.

21 Mutirangura A, Ledbetter SA, Kuwano A, et al. Dinucleotide repeat polymorphism at the $\mathrm{GABA}_{\mathrm{A}}$ receptor $\beta 3$ (GABRB3) locus in the Angelman/Prader-Willi region (AS/PWS) of chromosome 15. Hum Mol Genet 1992;1:1.

22 Lindeman $R$, Kouts $S$, Woodage $T$, et al. Dinucleotide repeat polymorphism of D15S10 in the Prader-Willi chromosome region (PWCR). Nucleic Acids Res 1992;19:5449.

23 Lathrop GM, Lalouel JM. Easy calculations of lod scores and genetic risks on small computers. Am $\mathcal{f}$ Hum Genet 1984;36:460-5.

24 Knoll JHM, Glatt KA, Nicholls RD, et al. Chromosome 15 uniparental disomy is not frequent in Angelman syndrome. Am f Hum Genet 1991;48:16-21.

25 Hall JG. How imprinting is relevant to human disease. Development (suppl) 1990:141-8.

26 Heutink P, van der Mey AGL, Sandkuijl LA, et al. A gene subject to genomic imprinting and responsible for hereditary paragangliomas maps to chromosome 11q23-qter. Hum Mol Genet 1992;1:7-10. 